\title{
REPERCUSIONES ACADÉMICAS Y SOCIALES DE LA ENSEÑANZA DE LA MEDIACIÓN EN LA FORMACIÓN DEL ALUMNO UNIVERSITARIO
}

\author{
SOCIAL AND ACADEMIC REPERCUSSIONS ABOUT LEARNING MEDIATION \\ IN THE FORMAL BACKGROUND ON HIGHER EDUCATION
}

\section{Resumen}

as necesidades de la sociedad respecto a las relaciones nos han obligado a establecer nuevas formas de solución de conflictos de manera pacífica, amigable, cercana y fuera de las instancias gubernamentales. Por esto, es necesario reforzar una enseñanza de los métodos autocompositivos desde la formación de los futuros profesionistas a través de una revisión de las opciones y consecuencias de la enseñanza y aplicación de los mecanismos alternativos de solución de conflictos (comúnmente conocidos y así se denominarán en el presente artículo, por sus iniciales como MASC) en las aulas, no únicamente para la comunidad escolar, si no de todas las instituciones socializadoras, desde la perspectiva académica, legal y social.

Palabras clave: mecanismos alternativos de solución de conflictos; formación profesional; planeación de la educación; universidad; transformación social.

Abstract
lutions beed in society about interpersonal relationships, have made us stablish new ways of conflict reso-
cial jurisdiction; therefore is necessary and important to strengthen the autocompositive mechanisms in higher education, through options and consequences of teaching the alternative dispute resolutions, not only to use them in the school community, but in all institutions, from academic, legal and social perspective.

Key words: alternative dispute resolutions; professional education; educational planning; university; social transformation.

1 Licenciada en Derecho por la Universidad Autónoma de Ciudad Juárez. Estudiante de la Maestría en Negociación y Mediación en el Instituto de Mediación de México S.C., México. Correo electrónico: marisol.borrego@hotmail.com. ORCID: 0000-0002-6928-5572. 


\section{Introducción}

Las necesidades de la sociedad han ido cambiando de acuerdo con diversos procesos evolutivos en el transcurso del tiempo, tanto biológicos como sociológicos, y con ello, sus formas de aprendizaje, razonamientos, posturas, ideales y formas de interacción, siendo entonces que las leyes han ido modificándose para la satisfacción de los mismos.

Es por ello que, siendo tan importante el proceso educativo, se pretende explicar e impulsar la urgencia de que, quien se encuentre realizando sus estudios superiores, tenga conocimiento de los MASC, a partir de los modelos de enseñanza que se han enfocado en favor del alumnado, en este caso, refiriéndose a quienes se encuentran en estudios profesionales y de posgrado, y con ello, permitir relaciones laborales exitosas, ya que en ellas se invierte por persona, por lo menos seis y hasta doce horas diarias o más, siendo casi imposible evitar el conflicto, al menos de información y relación, si tomamos en cuenta que, todos formamos parte de entornos laborales, además de la plusvalía del trabajador frente a otros que no cuenten con estas herramientas.

\section{Planteamiento}

La mediación en México tiene relativamente poco tiempo de encontrarse establecida en la legislación. Tanto constitucional como localmente, a raíz de las reformas del 2011, donde se vio privilegiado el acceso a los derechos humanos de las personas, específicamente con la ponderación de la solución de conflictos sobre cualquier formalismo procedimental como lo cita la Constitución Política de los Estados Unidos Mexicanos, en la adición del artículo 17 realizado el quince de septiembre del 2017, (tomando en cuenta que la Carta Magna tiene 103 años 
de publicada). En el estado de Chihuahua se introdujo a través de la Ley de Mediación publicada en 2003 por el Periódico Oficial del Estado de Chihuahua, la cual fue derogada sin alcanzar a ser aplicada, pero en su lugar emitida y publicada la Ley de Justicia Alternativa del Estado, y así en el Periódico Oficial del Estado de Chihuahua el treinta de mayo del dos mil quince, y vigente a la fecha. Esto genera entonces imperantemente, que su conocimiento se realice desde el ámbito académico, a fin de generar una nueva realidad en la que se privilegie el diálogo y que a largo plazo impacte psicológica, cultural y sociológicamente, en la comunidad, ya que hasta ahora, se ha aplicado paulatina y lentamente, con las reservas de los juzgadores, así como el general de la sociedad que desconoce del tema, e incluso de los propios mediadores, así como su poca instrucción en el ámbito académico.

Para lograr que la mediación y en general, todos los MASC sean aplicados en las relaciones personales y laborales se deben implementar y promocionar desde los centros educativos, como herramientas para la vida, de manera común y simple. Siendo indispensable que sea una parte de la instrucción en la universidad, por ser donde se adquieren las habilidades específicas para el desempeño de su profesión a manera de especialización y sobre todo, las formas de resolver los problemas mediante instrumentos utilizados y validados durante el propio crecimiento y desarrollo humano. Es decir, que en la vida diaria se debe tener un mínimo autoconocimiento y autocontrol sobre emociones, eliminando el aspecto adverso del conflicto, que generalmente se encuentra. Como Ortuño lo enuncia (2014): "ligado a connotaciones negativas, sentimientos y experiencias desagradables de hostilidad y malestar; a menudo confundido con conductas violentas con consecuencias sancionadoras, tras la reflexión y conocimiento de su verdadero significado, experimenta un cambio favorable hacia la connotación positiva del término" (p. 327).

La importancia de generar conciencia respecto a la instrucción de los futuros profesionistas mediante una cercanía natural a la resolución pacífica de conflictos, permitirá reproducir la autocomposición en la toma de decisiones además de una naturalización de ello ante la comunidad. Desde la eficacia en el tiempo que se invierte, la duración o permanencia de los efectos en sus resultados al encontrar satisfacción de todos los intervinientes, y la comprensión de emociones de cada involucrado, siendo en el ámbito privado hacia sus clientes y en el ámbito público hacia los ciudadanos, evitando como lo cita Carolina Macho Gómez en su estudio doctoral, "la paulatina formación de esta cultura litigiosa en la ciudadanía, la intensa actividad reguladora de los Estados, junto con el desarrollo social, cultural y econó- 
mico alcanzado en los últimos tiempos, ha provocado la llamada jurisdiccionalización o hiperjudicialización de las sociedades occidentales contemporáneas" (2014, p. 934).

Y continúa explicando, "[e]s evidente que esta multiplicación de causas planteadas ante los órganos jurisdiccionales no ha podido ser manejada, ni gestionada apropiadamente, por el método clásico: el proceso judicial". Con ello, entenderemos que es trascendental entonces, buscar nuevas formas de comunicación formal, es decir, que tenga las mismas consecuencias jurídicas y efectividad que una sentencia o que la resolución judicial aporta; y que conlleva previamente y de manera indispensable, a su estudio a nivel educativo superior, a fin de evitar su aplicación improvisada.

La búsqueda de la cultura de paz se debe entender como lo establece en la definición de la Organización de las Naciones Unidas (ONU, 1999) en el artículo primero de la Declaración y Programa de Acción sobre una Cultura de Paz en la resolución A-RES/53/243 aprobada por su asamblea general: "La cultura de paz consiste en una serie de valores, actitudes y comportamientos que rechazan la violencia y previenen los conflictos tratando de atacar sus causas para solucionar los problemas mediante el diálogo y la negociación entre las personas, los grupos y las naciones" (p. 3).

En el documento citado, la ONU toma en cuenta la historia mundial, refiriéndose a que los países dejaron de lado la cultura de la paz para la resolución de conflictos, con consecuencias y afectaciones catastróficas: como el holocausto con miles de pérdidas de vidas humanas; desastres ambientales como Hiroshima, desabasto de recursos naturales o la separación social, que es la principal generadora de violencia.

Regresando a la implementación de la mediación como parte de la formación del alumno universitario, es necesario de acuerdo con Grau, González y Álvarez (2016) "ofrecer al alumnado una orientación personal y apoyo a su desarrollo académico, personal y en la adaptación al contexto curricular y social universitario" (p. 366), estableciendo de alguna manera el contacto primario a la diversidad en cuanto a resolución pacífica de conflictos, así como un impulso al interés genuino, y con ello, el cambio de canon social, tomando ventaja también de los vertiginosos cambios sociales que las nuevas tecnologías ofrecen.

Ortuño (2014) explica exactamente la necesidad de los alumnos sobre adquirir este conocimiento en el ámbito educativo en la universidad, debido a que "saber afrontar un conflicto con alumnos, compañeros, familiares, es uno de los beneficios más claros que la mediación puede aportar a todos los componentes de la comunidad educativa, puesto que este aprendizaje, trascenderá a situaciones futuras” (p. 17). 
Como consecuencia, generará a mediano plazo las posibles modificaciones conductuales, para que sean visibilizados los beneficios y la importancia de la mediación también en las otras instituciones socializadoras: familia y comunidad, retomando las palabras de Ortuño "al hablar de las ventajas de la mediación, Suáres (1996, p. 53), hace referencia al concepto de deuteroaprendizaje y así recoge: $\mathrm{Al}$ solucionar un conflicto, como subproducto de esto uno puede adquirir la capacidad de solucionar otros futuros conflictos en la misma área en la cual se presentó el anterior o aun en otras áreas diferentes” (2014, p. 17).

La adquisición del conocimiento desde la escuela como una institución socializadora influirá como apoyo para la difusión e incorporación de los mecanismos alternativos de solución de conflictos a la vida diaria: cubriendo las consecuencias de laborar fuera de casa de ambos padres, abuelos que cuidan de los nietos en el mejor caso, así como guarderías o escuelas de tiempo completo que han intentado suplir algunas deficiencias o carencias en cuanto a la educación inicial, incluyendo el proceso comunicativo y hasta la inteligencia emocional se han rezagado por la comunidad, dejándolo como una simple materia en la educación básica, por lo que al llegar a un nivel universitario, los alumnos carecen de esas habilidades cognitivas, comunicacionales y de interacción, además de la exi- gencia a la institución educativa, que se ha tornado de formación a transformación.

\section{La mediación en México, el estado de Chihuahua, y la legislación vigente}

La participación del Estado en el proceso de resolución de los conflictos durante los últimos años ha reflejado el alejamiento a las necesidades del individuo al momento de la creación y modificación, así como de interpretación y aplicación de la ley, tanto por los legisladores como por juzgadores, como lo afirma Cabello Tijerina (2013): "Conforme las sociedades fueron adquiriendo mayor complejidad, el uso y la práctica de las vías de pacificación social como la mediación se vieron mermadas hasta casi desaparecer; recayendo la responsabilidad de la impartición de la justicia en el Estado, quien monopolizó la resolución de los conflictos a través del procedimiento judicial, repercutiendo en su sobresaturación y excediendo su capacidad resolutoria, contrariando así las características de prontitud que debe tener la justicia” (p. 193).

Sin embargo, la misma legislación en México ha dejado una ventana abierta a la mediación a partir de los movimientos sociales, desde un nivel constitucional, como lo fue el artículo 156 de la Constitución Política de los Estados Unidos Méxicanos del año 1824, en donde se estableció: "A nadie podrá privarse su derecho de terminar sus diferencias por medio de jueces 
árbitos, nombrados por ambas partes, sea cual fuere el estdo del juicio", mismos que han hecho a la comunidad replantearse en los diversos momentos, de las conductas encuadradas en sus leyes, además de la obligatoriedad actual de ajustarse a las normas internacionales, ya que desde los organismos como Unicef (Fondo de las Naciones Unidas para la Infancia, por sus siglas en inglés) y la ONU han incorporado en sus estatutos, estudios e investigaciones, así como en sus diálogos y postura a la mediación y los MASC como pilares de las nuevas estructuras y planteamientos, para cambiar paradigmas, como se ha hecho en los diversos conflictos, y como lo hicieron en su momento Fisher y Ury en los conflictos internacionales con lo que perfeccionaron la teoría de Harvard.

El cambio de prácticas y las condiciones sociales deviene en los últimos tiempos; como Herrera Heredia (2008) explica, desde "La propia revolución mexicana, como movimiento social, propició el florecimiento de principios como el de justicia social y sentó las bases de un derecho laboral, que quedó plasmado en el rango constitucional en el artículo 123 de nuestra Carta Magna de 1917; ello motivó (la creación de) la Ley del Trabajo del Estado de Veracruz, de 14 de enero de 1918, primera en su tipo en el país, y generó posteriormente la Ley Federal del Trabajo del 18 de agosto de 1931 que, conforme a dicho principio es el marco que regula la protección de los derechos de los trabajadores, así como los procedimientos para solucionar las diferencias que surjan entre el patrón y el trabajador" (p. 1), y que, analizando su cumplimiento en la actualidad, de lo global hacia lo particular de cada comunidad, se han encontrado respuestas en los mecanismos a la protección de la voluntad, independencia y libertad de las personas, así como el cumplimiento a las indicaciones a las que el Estado mexicano se debe adherir, tomando en cuenta las consideraciones según la Carta de las Naciones Unidas sobre el mantenimiento de la paz y la seguridad internacionales $(4658,1991)$ los Estados miembro "harán todos los esfuerzos posibles para lograr el arreglo pacífico de las controversias de carácter local por medio de tales acuerdos u organismos regionales antes de someterlas al Consejo de Seguridad".

La sociedad ha crecido con la creencia de que el acceso a la justicia se da a través de una autoridad que castigue y corrija al otro, desde la aceptación de responsabilidad, actitudes, comportamientos y formas de interactuar, por lo que "la costumbre y tradicionalismos de judicializar todos los conflictos, por pequeños o grandes, simples o complejos que fueran, fue agudizando la necesidad de pensar en otros métodos para resolverlos (desde la mediación, conciliación, arbitraje, negociación y justicia restaurativa, entre otros) para despresurizar el sistema judicial; sin embargo, "refie- 
re (Munguía, 1999) la manera desordenada como hasta ahora han sido utilizados los MASC, explica la confusión que existe sobre su significado y la peculiaridad de cada una de ellos. Además, que la participación del Estado ha sido incipiente en promocionar estos métodos para solucionar los conflictos" (Cornelio, 2014, p. 87).

Junto con el reconocimiento de los MASC como un derecho de acceso a la justicia a nivel constitucional en el año dos mil diecisiete, cada Estado se vio obligado a reformar su legislación, y adecuarla a la nueva realidad vivida. Así lo menciona Fernández Cuevas (2017), que a partir de estas reformas constitucionales "la mitad de las entidades federativas que lo conforman (al país) han desarrollado mecanismos alternativos para la solución de controversias, con la finalidad de impulsar reformas legales y procesales e inclusive se han creado centros en exprofeso para la impartición de la justicia alternativa”.

\section{La mediación en las instituciones}

El conflicto, visto desde cada una de las instituciones judiciales, académicas y sociales, como lo plantea Herrera (2008), establece que la mediación "es producir un aflojamiento de la tensión existente entre las partes, para llevarlas a una buena comunicación, un enfoque futuro y con un resultado en que ambos ganan. Mientras que, en las cuestiones controvertidas, el enfoque es hacia el pasado, donde la ten- sión continua y una parte gana y la otra pierde" (p. 9); ello permite la concientización y toma de responsabilidad de cada persona involucrada en el conflicto: dar autonomía de gestión y de negociación a las partes, permite el aumento de valores y la adquisición de responsabilidad de las emociones sentimiento de justicia y generación de soluciones más efectivas para cada interviniente, dando a la ley, el marco de actuación y el punto de partida del diálogo, pero a la justicia el valor central a través de la comunicación, bajo una disciplina conductual y de respeto, desde un ejercicio íntimo e integrador, en el cual las personas en conjunto miran hacia la misma dirección en busca de la solución.

\section{La mediación actualmente dentro de las universidades en México}

La familia como institucion socializadora ha limitado su trabajo en la educación emocional por circunstancias economicas, sociales, culturales y académicas, dejando que sea la institución educativa, la institución formadora, misma que ha privilegiado la educación punitiva y coercitiva, es decir, del castigo a determinadas acciones "antisociales, contra las leyes y costumbres", tal como lo ha hecho el Estado a través de las leyes, dejando de lado o hasta ignorando la educación para la vida, enfocados únicamente en la mayor especialización en las ramas de estudio, Ortuño (2014, citando a Pantoja, 2005), quien postula que "en el 
marco de la educación obligatoria, los conflictos constituyen una de las preocupaciones más importantes de docentes, tutores, orientadores, padres e instituciones educativas. El respeto y el mantenimiento del orden en nuestras aulas resultan actividades a las que se dedica a diario un gran esfuerzo sin que se consigan, en muchos casos, los resultados esperados" (p. 126).

Así también el artículo 3 fracción II inciso c) de la Constitución de 1917, con las reformas de 2012, determinó que la educación "contribuirá a la mejor convivencia humana, a fin de fortalecer el aprecio y respeto por la diversidad cultural, la dignidad de la persona, la integridad de la familia, la convicción del interés general de la sociedad, los ideales de fraternidad e igualdad de derechos de todos". De la tal suerte que Chihuahua no fue la excepción con la publicación de la Ley de Justicia Alternativa del Estado, y su principal objetivo de cambiar costumbres del litigio de una sociedad en la que impera la confrontación, por una en la cual sus integrantes aprendan a colaborar y a dialogar, como se establece desde el artículo primero de la misma ley (2015, art. 1): "Fomentar y difundir la cultura de paz y de restauración de relaciones interpersonales y sociales".

Para el docente, resulta una tarea adicional la de construir un orden, y no simplemente jerárquico, sino de respeto, inteligencia, sapiencia y guía, como un parteaguas en el conocimiento, ya que ahora, también se esfuerza en encontrar las necesidades primarias y de descubrimiento de emociones del alumnado, además de ser tutores, ahora también son consejeros, representantes y hasta investigadores que deben verificar si la educación en casa es la suficiente, si las necesidades primarias están siendo cubiertas o si hay algún delito que perseguir.

Es por ello que se tiene como objetivo el de transformar la manera de resolución de conflictos de las personas desde la etapa de preparación académica, y con ello, encontrar nuevas maneras de desarrollar conciencias y personalidad, generando el impacto en las personas cuando sean empleados o empleadores y que, dentro de las relaciones laborales, modificaran las actuales comunidades.

Para las organizaciones internacionales como Unicef y la Organización Internacional del Trabajo (OIT), las situaciones de conflicto se relacionan directamente con el alcance de objetivos, tomando en cuenta los valores otorgados para cristalizar las posibles soluciones, a fin de alcanzar los mayores beneficios a través del diálogo mediante puentes de comunicación eficientes, lo que puede pensarse sencillo; sin embargo, lograr un desarrollo efectivo y conclusión positiva en favor de los intervinientes privilegiando el ambiente laboral, es una necesidad y requerimiento de los empleadores hoy en día, que es dificil encuadrar en una ley, sin la capacitación 
mínima de sus teorías, beneficios, principios y forma de llevarse a cabo.

También es necesario tomar en cuenta las limitantes del estudiante, en cuanto a los antecedentes familiares, culturales y hasta psicológicos y neuronales de cada uno, porque también dependerá de estos enfoques el resultado que se obtenga, es decir, su trascendencia será conforme el proceso mental y de actitud que el propio estudiante imprima en su educación; sin embargo, también se deberán tomar en cuenta los conocimientos previos que el docente tenga, y que este crea en esta necesidad de cambio, y por ende tenga también mas probabilidades de contagiar de esta necesidad a sus estudiantes, contrario de un docente que solo aplique de manera teórica los conocimientos y pueda o quiera resolver las dudas de manera efectiva y consciente. Las competencias pedagógicas del docente tendrán gran relevancia al momento de institucionalizar la mediación dentro del contexto del proceso educativo como natural: "Podríamos definir la institucionalización como el proceso por el cual una innovación encaja o se integra en los procedimientos operativos de un sistema. Dicho de otra manera, la institucionalización es cuestión de la extensión con la que los usuarios internos (del aula a las Consejerías y Ministerio de Educación) aceptan y usan una innovación de manera cotidiana" (Ramón Alzate, 2010, p. 8). La experiencia de la implemen- tación del sistema penal acusatorio o conocido como Sistema de Justicia Oral, en el que primordialmente se actualizaron o modificaron algunos conceptos, como el principio de presunción de inocencia y la forma de llevarse a cabo los juicios, así como la adopción de los MASC para la resolución de asuntos, permite prever que el proceso de implementación será difícil si no se dan a conocer los principales actores de manera previa, desde todas las aristas posibles, beneficios, y entendimiento en el tema educativo para que de manera gradual, pueda ser implementado.

Tomando como ejemplo el ámbito educativo en España, según Ortuño (2014), desde el siglo XX surge la preocupación por el concepto convivencia escolar, y aparece por primera vez en la Ley General de Educación de 1970 de dicho país (p. 14), tomándose en cuenta como parte primordial, después de hacer conciencia en las repercusiones sociales y de desarrollo personal y académico de omitir la generación de ambientes pacíficos desde la comunidad académica.

En México a nivel educativo, se han tomado medidas para la regulación de mediación escolar en todos los niveles educativos a través de políticas públicas que tomaron fuerza en el 2015, con el Programa Nacional de Convivencia Escolar de la Secretaría de Educación Pública (SEP), estableciendo en su momento, de acuerdo con la exposición de motivos de su publi- 
cación, una normatividad, aunque mínima, para la "garantía de los aprendizajes como tal, más la convivencia no violenta, apegada a los derechos humanos", pero que hasta ahora no se ha determinado una línea de acción, tomando en cuenta con la literalidad o importancia y fuerza necesaria dentro de la enseñanza formal hacia los estudiantes en ninguno de los niveles educativos, si se toma en consideración que no hay en las instituciones, ni las estructuras formales con el personal preparado, especializado y familiarizado con la cultura de paz, ni programas de formación de mediadores pares, consejo o equipo de mediación especializado de la que puedan echar mano los docentes, en las secretarías de los Estados, o en la SEP.

Tal como lo explica Sonia Paris (2005) en su tesis doctoral: "la educación tiene un papel importante en aquellos lugares en los que, tradicionalmente los conflictos han sido regulados negativamente con la finalidad de cambiar estas actitudes e introducir reacciones pacíficas” (p. 91), estableciendo la importancia de la enseñanza a través de las instituciones formadoras, que permiten realizar cambios sustanciales de paradigmas sociales mediante la normalización y ponderación del diálogo, la empatía, enfoque en el conflicto y no en la persona.

A su vez, Inciarte, Romero y González detallan que "en el ámbito educativo, Monjo (1999, citado por Picón y otros, 2005); define la mediación como "una forma alter- nativa de resolución de conflictos hasta llegar a una solución consensuada, satisfactoria y mutuamente aceptada” (p. 46). Partiendo de este concepto, el autor señala que la mediación escolar implica un modelo de ayuda para analizar y resolver sus conflictos desde perspectivas constructivistas, atentos y respetuosos de los sentimientos e intereses de los otros; pero, sobre todo, constituye un importante trabajo preventivo y formativo, tanto a nivel individual como colectivo, explicado de mejor manera por Pesqueira y Aub, en el sentido que:

En los nuevos y diversos modelos de familia, nacen niños quienes desde su nacimiento tienen derecho a un ambiente favorable para iniciar su proceso de socialización. Pese a ello, los padres o tutores como agentes responsables de su cuidado y nutrición material y emocional, lamentablemente no ejercen esa responsabilidad más allá de lo mínimo, o sencillamente delegan esa responsabilidad al ámbito social. En estos casos los sistemas socializadores en ambientes escolar y comunitario se encuentran con personas con carencias formativas, dándose a la tarea y encargo de nutrir y desarrollar lo mejor posible en ellos aquellas conductas, hábitos y habilidades sociales aprobadas en esos contextos. Cuando la familia nutre y desarrolla al ser humano, la escuela y el barrio se 
encargarán de nutrir y desarrollar al ser social (Pesqueira y Aub, 2010, p. 156).

Es indispensable que toda la sociedad entienda la importancia de la enseñanza de la mediación como método para conseguir la transformación cultural de actitudes, posiciones, contextos, visiones, tomando en cuenta como definición de cultura la que cita Paris (2005) sobre la definición elaborada por Taylor (Cabedo Manuel, 2001, p. 24), "como sistema de concepciones transmitidas históricamente y expresadas en formas simbólicas por medios con los cuales los seres humanos comunican, perpetúan y desarrollan sus conocimientos y actitudes frente a la vida” (p. 92).

Las percepciones de las instituciones judiciales y sociales, poco a poco, han insertado en sus legislaciones, estatutos y la costumbre, la influencia de privilegiar los mecanismos ante cualquier formalismo procedimental, como necesidad de mejorar las relaciones interpersonales e impulsar la cultura de paz, y con ellos, obliga a las instituciones educativas, a actualizarse o evolucionar en dichos procesos.

Dentro del sistema judicial ya se encuentra agotado el tema de su pertinencia y su viabilidad, ya que las leyes, tanto la constitución, códigos y reglamentos han establecido claramente las opciones para su aplicación, así como los casos de excepción. Sin embargo, ante las instituciones educativas, ha sido más lento el proceso de aplicación y regulación, ya que hasta el momento no ha sido obligatoria su impartición, o al menos un acercamiento mínimo de todos los universitarios (exceptuando la carrera de derecho en algunas casas de estudios superiores), limitándoles hasta la fecha, de una formación básica apropiada respecto a la resolución de conflictos, incluso en ninguna etapa educativa se ha implementado de manera fuerte, efectiva y con un seguimiento adecuado, además de no ser tomados en cuenta como una necesidad social.

\section{Componentes de la comunidad edu- cativa como base de la enseñanza de la mediación}

Para lograr la modificación de conductas que la escuela permite, como lo cita Otuño (2014) a partir de "[s]aber afrontar un conflicto con alumnos, compañeros, familiares, es uno de los beneficios más claros que la mediación puede aportar a todos los componentes de la comunidad educativa, puesto que este aprendizaje, trascenderá a situaciones futuras" (p. 17).

De acuerdo con la organización de las Naciones Unidas en su Declaración y programa de acción sobre una cultura de paz, señala como las medidas para promover una cultura de paz por medio de la educación: "g) Reforzar las actividades en marcha de las entidades pertinentes del sistema de las Naciones Unidas destinadas a impartir capacitación y educación, cuando corres- 
ponda, en las esferas de la prevención de los conflictos y la gestión de las crisis, el arreglo pacífico de las controversias y la consolidación de la paz después de los conflictos" (ONU, 1999, p. 7), siendo la escuela, por excelencia en cualquiera de sus etapas, formadora de carácter y principios, enfatizando un equilibrio entre lo prohibido y lo esperado, es necesario que todos los componentes sociales, físicos o humanos y ambientales sean armonizados para la estructuración de la mediación como parte de la enseñanza formal de las personas futuras profesionistas, como potenciales líderes en las labores económicas del país.

Las instituciones educativas además deben impulsar la cultura de paz, a través de procesos analíticos y hasta de conveniencia social, es decir, desde la deconstrucción y modificación de relaciones y estructuras sociales preestablecidas, mediante su uso en las experiencias personales, hacia dentro de la institución como hacia el exterior, visibilizando la cultura del diálogo y entretejiendo nuevas redes de habilidades.

El docente como parte de este proceso es quien puede y debe impulsar el uso de los mecanismos alternativos de solución de controversias y hacer llegar el conocimiento a las y los estudiantes, de una manera digerible, así como detectar e impulsar perfiles de nuevos mediadores, y la posibilidad de fortalecer las habilidades comunicacionales de la comunidad estu- diantil, que finalmente pueden ser parte de un proceso.

Los estudiantes van a entender con el uso de ejercicios el proceso dialógico y su pertinencia, para que de manera libre puedan elegir sobre un proceso judicial, administrativo y heterocompositivo, la preferencia de un proceso de mediación autocompositivo, con conciencia de sus alcances, a través de una valoración consiente y detección de intereses y necesidades.

La comunidad con el rol de apoyo puedae afianzar la toma de decisiones de las personas como algo positivo, que empodera al individuo como un ente que puede resolver que en sus manos se encuentran también las soluciones, desarrollo de nuevas perspectivas, a través de la creación de entornos seguros, que propician e impulsan ambientes externos simétricos en el tema de la voluntad, compromiso y respeto.

\section{Diferencias entre programa de me- diación, la orientación escolar y enseñan- za de la mediación como habilidad}

Junco, (2010) explica que "[e]n los últimos años, los conflictos en la escuela han sido un estudio prioritario. Lo que ha causado diferentes formas de tratar esos conflictos, con consecuencias bastante efectivas" (p. 2), cambios realizados a través de un rol activo de la mediación incorporado en sus clases, las habilidades para la resolución de conflictos y la capacidad de transfor- 
mación de experiencias basadas en valores y convivencia.

La mediación en lo general, y la denominada escolar o entre pares en la universidad, que es la que se realiza en la institución educativa, a fin de que sean los alumnos los que resuelvan los conflictos entre ellos, requiere de los principios generales e inamovibles para su realización: voluntariedad, confidencialidad, flexibilidad, economía, etcétera; a diferencia de la orientación escolar, que deriva de la necesidad de los alumnos a la guía de sus propios conflictos hacia su interior, un trabajo hacia al interior para la interpretación de sus propios ideales. Por otro lado, la enseñanza de la mediación de manera formal como herramienta de conocimiento para el estudiante, nos permite en cierta medida, formar mediadores para la comunidad, hacia sus propios grupos laborales, así como sociales y familiares, que entiendan, acepten y difundan como alternativa y su socialización como modo de vida.

Rosa María Caycedo y Nancy Cocunubo (2016) toman en cuenta que "la violencia interpersonal o intergrupal se puede dar en cualquier contexto interactivo en el que intervengan seres humanos, como individuos o como grupos. Así, los ámbitos familiar, escolar, laboral, lúdico, deportivo o institucional son marcos psicosociales en los que pueden surgir estas conductas" ( $p$. 1734), generando en la institución escolar una parte fundamental para modificar o reforzar la costumbre social. La intención de la mediación como un proceso formativo de la comunidad permite educar al alumnado y no a sancionar la persona adulta, que pondere los mecanismos alternativos de solución de conflictos en la vida diaria.

Además, la educación universitaria tiene la finalidad de brindar herramientas para la vida y función de la persona como profesionista, y con ello, plusvalía sobre cualquier otro competidor en el mundo laboral, por lo que, el desarrollo de habilidades para la resolución de conflictos de manera pacífica y efectiva, genera un talento y valor añadido para superar los retos que se le enfrentan y ser mucho mas competente laboralmente, desarrollo de liderazgos y por ende generación de oportunidades.

Dentro de las grandes corporaciones de reclutadores de mano de obra y de desarrollo de competencias, así como de expertos en el área de recursos humanos, se detalla que la resolución de conflictos dentro del área laboral, es uno de los valores añadidos a las personas al momento de la contratación y asenso, tomando en cuenta que los conflictos tanto fuera como dentro de los centros de trabajo, son factores importantes para la reducción de la producción y/o eficiencia del mismo de manera invisible, pérdidas económicas y el conocido burnout o síndrome de agotamiento laboral, que puede ser transversal, lineal o mixto, que a pesar de estar regulado al me- 
Figura 1. Gráfica de la diferencia entre la materia, la mediación como servicio y la orientación escolar

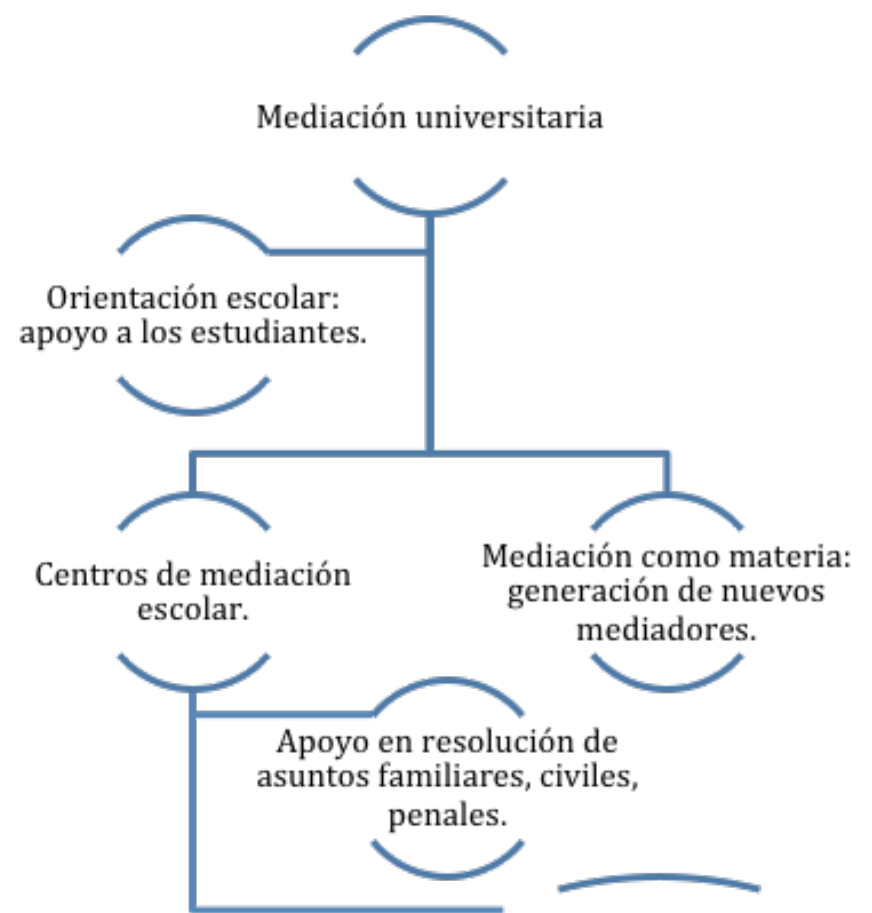

Canalización: en caso de no ser mediable (violencia de género, se afecten derechos de terceros, intereses de menores e incapaces ni cuestiones de orden público).

Fuente: Elaboración propia.

nos en la Ley Federal del Trabajo mexicana, aunque no directamente, si sus consecuencias, como posible causal de rescisión de contrato, siendo que su determinación queda al arbitrio de quien pueda tomar las decisiones de los departamentos de recursos humanos, patrón o la autoridad de lo laboral y no de quienes puedan ser parte del conflicto.

Es importante reconocer que los alumnos que egresan actualmente carecen de las habilidades sociales, por parte de los núcleos familiares y que las instituciones educativas en todas sus etapas, han ignorado en las planeaciones escolares, la impartición o desarrollo de estas capacidades e interaccionar de mejor manera con los otros que nos rodean.

Como los describe el Banco de Desarrollo de América Latina en el reporte de economía y desarrollo 2016,

Las habilidades son los saberes, hábitos y atributos de la personalidad que contribuyen no solo a la productividad laboral sino también a tener relaciones armoniosas con conocidos y desconocidos, a proponerse objetivos ambiciosos y diseñar planes cumplibles para alcanzarlos, a que los ciudadanos participen 
en la vida cívica de una manera más frecuente y comprometida, y a mantener conductas saludables, entre otros aspectos del comportamiento que hacen al bienestar de las personas. Pero los beneficios de las habilidades no se restringen al plano individual. A nivel social, una población con más habilidades podrá coordinar más fácilmente las acciones necesarias para la provisión de mejores bienes y servicios públicos, controlar mejor las amenazas a la salud pública (epidemias, pandemias, por ejemplo) y tener menores niveles de conflicto interno. Es decir, las habilidades individuales imponen externalidades positivas al resto de la sociedad (p. 21).

Que el estudiante de trabaje con los mecanismos alternativos de solución de controversias conlleva a la facilidad de que cuando se convierte en profesionista tenga la confianza de trabajar sobre cualquier situación, por cambiante que esta sea, ya que a través de la historia se refleja nuestro temor a lo desconocido y nos preocupan los cambios, por lo que la enseñanza, llevará un proceso de desaprender lo anteriormente adquirido y rehacer el conocimiento, forzando al cambio de paradigmas y estas antiguas tradiciones o herencia del litigio, de la no comunicación.

\section{Implicaciones de la enseñanza de los mecanismos alternativos de solución de conflictos por área de estudio}

La enseñanza no debe ser únicamente una potestad, facultad o beneficio de los estudiantes de derecho, ya que no son los únicos que manejan conflictos en su vida diaria; David Rodríguez (2017) plantea acertadamente, que "la mediación es una herramienta al servicio de la convivencia pacífica, que abre paso a una nueva cultura basada en el diálogo como estrategia de transformación de los conflictos. Su potencial para generar aprendizaje en cualquier persona por el hecho de participar en un proceso de mediación es evidente y, por ello, parece importante su extensión a todos los ámbitos de interrelación humana (familiar, penal, comunitario, intercultural, empresarial, político, etcétera)" (p. 40) dando paso a las necesidades entonces de su enseñanza de manera formal y estructurada, si deseamos que sea aceptada y utilizada comúnmente, previo a cualquier otra resolución posible.

El planteamiento hacia las futuras generaciones de empresarios, funcionarios y cualquier tipo de empleado, tiene su raíz y necesidad inminente de aplicación de acuerdo con Egla Cornelio (2014) respecto que "los conflictos son situaciones que se presentan a las personas en todos los tipos de relaciones, de manera que en las relaciones laborales también se encuentran pre- 
sentes, con frecuencia, porque los centros de trabajos son espacios donde convergen diversidad de personas, con intereses, formaciones, culturas, costumbres y valores. Pero también, los conflictos son oportunidades para transformar relaciones, modificar conductas o visto de otra manera, son oportunidades de crecimiento" (p. 94).

Por lo anterior, podemos aseverar que todo aquel que desea realizar y tomar la mediación como un estilo de vida o al menos como una habilidad importante que permita desarrollar su labor de la mejor manera, puede realizarlo sin importar que tipo de profesionista sea, ya que las necesidades del alumno, van desde el entendimiento de su realidad, tanto el actual, como el futuro y aspiracional, interacción con las relaciones de poder (entendiéndose como las de supra-subordinación), mismas que se trasladarán a la convivencia social y moral, a través de estímulos que sean relacionados con el desempeño de su profesión.

Que cada institución educativa cuente con su propio centro de mediación, puede permitir, tanto a sus alumnos como a sus docentes, separar a la persona del conflicto y no relacionarlo directamente con el proceso educativo, si no como una posible consecuencia de la convivencia o la diferencia de ideas entre estudiante o entre estudiante y docentes, como una posibilidad de la interacción mas no como una consecuencia personal, misma que no tendría tampoco una relación sine qua non para la enseñanza de la mediación en las aulas como parte de su educación. Además, el hecho de apoyar la maduración de las habilidades psicosociales permiten reforzar el constructivismo educativo que las instituciones desean impulsar como parte de los modelos actuales de aprendizaje.

Como base de enseñanza de quien vaya a conocer de los MASC serán los principios para su aplicación siendo importante que, durante el estudio de la mediación como forma principal de solucionar conflictos en los ámbitos escolares, para su ejercicio hacia los empleos, familia y comunidad en general; si bien es cierto, es importante que los MASC se establezca su estudio desde temprana edad, es indispensable que el estudiante universitario aprenda a trasladarlo a cada una de las diversas actividades profesionales que realice.

Si durante la enseñanza de los mecanismos alternativos de solución de conflictos se logra que la empatía sea parte de su formación (Lungman, 1996), "sí pretendemos romper el ciclo de violencia y guerra, ya sea en un nivel interpersonal o internacional, debemos empezar por enseñar a nuestros estudiantes cómo tratar con los conflictos en forma no violenta. Debemos reemplazar una actitud adversarial (usted en contra de mí) por una perspectiva cooperativa de trabajo en conjunto para resolver problemas comunes" (p.12). Si es posible que la comunidad escolar sea un buen comienzo para la difusión e implementación en todas las 
áreas aledañas a la educación, tomando en cuenta que "el programa de mediación de estudiantes envía un importante mensaje: 'En esta escuela nosotros podemos hablar acerca de nuestras diferencias. No tenemos que pelear para obtener justicia', por lo que más y más estudiantes aprenderán a atacar al problema y no a la persona" (Lungman, 1996, p. 13).

Los temas de enseñanza, tal como lo mencionan Rosa María Caycedo y Nancy Cocunubo, "[p]or ningún motivo intentan desvirtuar el concepto de justicia y mucho menos hacer desaparecer los Tribunales, por el contrario, resultan ser una alternativa que auxilia y complementa la misión que hasta el momento han venido desempeñando" (p. 1740), lo que nos permite seguir brindando la certeza jurídica en cualquier ámbito de aplicación y las posibles consecuencias a la comisión de actor contrarios a la ley, que satisfagan en realidad las necesidades de los usuarios.

\section{Implicaciones sociales de su ense- ñanza en nivel universitario}

Las implicaciones de la enseñanza en la educación superior tiene una connotación de suma importancia para el cambio de paradigmas, como es citado por Pesqueira Leal \& Ortiz Aubb (2010), “[e]n la escuela, la violencia produce la disminución en el rendimiento escolar, la estigmatización de los estudiantes agresivos, quienes en consecuencia se reafirman como malos, la expulsión y el abandono de actividades escolares” (p. 23) estigmas con las que cargan durante su vida y que generan afectaciones psicológicas permanentes.

Si se realiza un entendimiento de las implicaciones y del porqué es necesario dar a conocer la mediación desde un proceso formativo, se toma en cuenta a Criado (2010) respecto a: “¿Y cómo lo hacemos? Con rigor, esfuerzo y profesionalidad. Es una profesión que se aprende, el mediador puede nacer, pero por regla general se debe hacer. Se hace con una formación de origen y recibiendo además una segunda formación rigurosa y especializada” (p. 6). Apoyándose en el cambio de estructuras mentales de los educandos, quienes realizan un proceso de selección y modificación de antiguos conocimiento para adquirir los nuevos.

Los procesos voluntarios y confidenciales que se realizan con la mediación, llegan a lo que David Rodríguez (2017) menciona en su tesis, como

La teoría de la incoercibilidad, tiene su impacto en la educación por medio de los valores y características personales que contraen obligaciones como lo son la buena fe, la probidad, la ética, la moral. La teoría incoercible propone al maestro y al directivo como a la comunidad educativa en general, la responsabilidad individual de resolver por mérito propio 
y no por leyes, reglamentos o normas los conflictos derivados entre ellos” (p. 233).

La naturalización de la violencia ha generado que las personas se aíslen en los problemas, necesidades y preocupaciones propias, generando un sentimiento colectivo de injusticia, incluso cuando no se haya necesidad de la intervención de una autoridad, traspasando fronteras y generaciones hasta llegar a la indispensable necesidad de respuestas, incluso aunque las tengamos al frente, imperando la ley del Talión (del más fuerte y de ojo por ojo), con una predominación de venganza, insatisfacción, que abre espacios de conflicto y no de diálogo, "es importante precisar que violencia y conflicto no son sinónimos, ya que un conflicto, tal como se planteó anteriormente, hace parte de lo que se vive cotidianamente en las relaciones sociales, pero no necesariamente trae como consecuencia la violencia. Por el contrario, y tal como se entiende en el presente estudio, violencia y agresión sí se entienden como sinónimos, dado que en ambas se advierte una intención específica de hacer daño y esta no es una variable inevitable de las relaciones" (Arias, 2017, p. 59).

La falta de estrategias desde la educación básica representa la carencia de la autoridad educativa por imponerse o resaltar los temas humanísticos, en el sentido que hasta ahora, las comunidades tanto educativas como laborales se han enfocado en las nuevas tecnologías, conectar a la gente que se encuentra lejos pero sin refuerzo de los lazos de los que se encuentran e interactúan de manera cotidiana; por esto, hay dos repercusiones de suma importancia: los posibles cambios académicos que se pudieran hacer y las implicaciones en la comunicación actual, desde los conflictos en los que se participan de acuerdo con la falta de información, de intereses, estructurales, de valores, de relación y ahora hasta de interpretación, sobre todo la digital.

La responsabilidad que cada individuo acepte respecto a un conflicto, permite a la sociedad fortalecer el entendimiento de la otredad, hacia el mejoramiento de la comunicación, "es de toral importancia que los conflictos sean abordados de manera adecuada y oportunamente, toda vez que, por el estado del tiempo los conflictos son emergentes, latentes y manifiestos. Si un conflicto se deja pasar y no se atiende a tiempo, la posible solución al mismo no tan solo puede ser compleja, sino también difícil”. Ello como consecuencia, impulsa los posibles desacuerdos a un proceso litigioso desgastante, incómodo y que en gran medida, no obtiene el resultado restaurativo deseado, como tampoco un cumplimiento de las necesidades sobre el conflicto con el cumplimiento de la sentencia, ya que el juez o tercero responsable, no se adentra ni en emociones, sentimientos, necesidades, hasta el simple hecho de ser 
escuchado de manera empática, únicamente encuadra la ley al caso concreto.

\section{$X$. Diferencias entre alumnos que reci- ben la instrucción}

Debemos estar conscientes de que, como lo menciona Jiménez Fernández (2008), "[l]a escuela, como organización social, tiene los mismos problemas que el resto de la sociedad: es un espejo que suele reflejar los males del entorno. Por lo tanto, como organización debe cuidar el propio entramado y orientar la acción hacia la prevención y prevención ante la aparición de conflictos perturbadores de la paz escolar" (p. 3).

Es necesario determinar, como lo conceptúa María Arias (2017), que los universitarios al término de su carrera deberán "precisar que violencia y conflicto no son sinónimos, ya que un conflicto, hace parte de lo que se vive cotidianamente en las relaciones sociales, pero no necesariamente trae como consecuencia la violencia” (p. 59), detectar los posibles generadores de violencia y transformados en oportunidades de mejora social.

Por lo que, si desde el inicio de la vida profesional se toma en cuenta la mediación como una parte de la formación académica, las realidades serán distintas, y así lo establece Juan Ortiz en su proyecto de intervención escolar, si además esta formación la realizamos conjuntamente con todos los sectores de la comunidad educativa habremos dado un paso de gigante en la mejora de la convivencia escolar, ya que desde un primer momento hacemos partícipes a todos y a todas en la gestión esa convivencia, y en la apuesta por un sistema de resolución no violenta de los conflictos, en base al diálogo y la colaboración (2016, p. 22).

Siendo sumamente necesario adentrarnos en la diversidad de la impartición de la educación a cualquier nivel (Aguirre, 2014): "reflexionar en el contexto socioeducativo que nos ocupa por ahora: deslocalización de saberes, territorios de la educación, mediación educativa, gestión de la diversidad, competencias digitales y enseñanza de la investigación” (p. 22) y con ello, las diversas consecuencias de su implementación.

Así es como Gloria Gómez Funes, nos permite avizorar a los profesionistas que ahora serían también mediadores en como pueden reducir y transformar las relaciones laborales,

siendo el conflicto algo inherente a las organizaciones, y el cambio una necesidad para la progresión de cualquier organización, se hace necesario que la organización desarrolle técnicas para la gestión proactiva y productiva del conflicto, propiciando en su seno la creación 
de espacios de aprendizaje individual que favorezcan procesos creativos que faciliten y propicien el desarrollo de las competencias requeridas, que motiven el crecimiento personal y la eficacia empresarial, permitiendo una adaptación adecuada al contexto cambiante en el que se desenvuelven, mediante el establecimiento de formas y procesos idóneos que propicien ese aprendizaje y ese cambio para, gradualmente, ir desviando el enfoque hacia un aprendizaje organizacional (Gómez Funes, 2013, p. 76).

Es necesario ver a las universidades como formadoras de personas, que definan valores, establezca reglas a través de la costumbre, "Las organizaciones modernas no solo son lugares de trabajo, sino que constituyen espacios de interacción y crecimiento personal (Vall, 2004) y en este sentido están formadas por personas que, como tales tienen necesidades que tienen tendencia a satisfacer dado que son requisitos de supervivencia (Henderson, 1971)" (Novel Martí, 2010, pág. 73), y como bien lo cita también Antonio Isaac Gómez Alcántara (2014) en su texto, la mediación es producto del detrimento de las estructuras jurídicas y sociales, "deterioro notable en el sistema jurídico, producto evidente de la multitud agobiante de juicio y demandas en todos los ámbitos de la administración de justicia, y de falta de ética en las relaciones laborales, y de toda índole” (p. 133).
Para que la mediación pueda ser tomada en serio, la sociedad y los responsables de la educación como función o sistema estatal, política pública, o necesidad social, deben tener como objetivo el de (Pérez Jiménez, 2016): "Contribuir al establecimiento de un Sistema de Mediación Escolar que posibilite la resolución pacífica de conflictos y que contribuya en la consolidación de una cultura de paz, con mayores niveles de empatía y aceptación de la diversidad” (p. 7).

Para que tengamos un pensamiento real sobre las acciones educativas, hay que analizar las necesidades de sus elementos: hablando de estudiantes, personal docente, administrativos, infraestructura e historias académicas alrededor, "En los centros educativos, las aulas presentan realidades heterogéneas, muy variadas, a veces complejas, también enriquecedoras, que hacen necesario este tipo de educación. Es básico educar en valores al individuo, educar para conseguir entornos inofensivos, de no violencia en sus diferentes manifestaciones, posibilitar que los sujetos aprendan también a solucionar situaciones diversas con otros, de forma conciliadora” (Silvia Pizarro, 2018, p. 209).

Que la sociedad y el propio Estado, así como las instituciones educativas consideren la mediación como una oportunidad para la resolución de los conflictos, incluso transformación de conductas desde un ámbito amigable, y en una etapa 
temprana, puede resolver muchos otros problemas sociales, ya que:

La mediación, como método de solución de conflictos aborda las controversias desde sus orígenes, cuando las conductas desarrolladas no constituyen delito alguno sino simples desavenencias vecinales, mismas que no son atendidas en los juzgados correspondientes, por tal motivo se corre el riesgo de que posiblemente se puedan convertir en un futuro en conflictos más severos y/o que puedan terminar en situaciones delictivas; lo cual se puede evitar y resolverse desde sus inicios por medio de la mediación para evitar así llegar a situaciones que empeoren la relación entre los individuos (Díaz Álvarez, 2016, p. 88).

\section{Conclusiones}

Antes de proponer, hemos dejado claro que la mediación busca transformar las relaciones dentro de todos los ámbitos: social, educativo, pero no menos importante y donde encontramos las consecuencias mas palpables, el laboral, descrito por Patricio Poblete (2012) en su tesis como "la necesidad de canalizar las relaciones laborales mediante un conjunto de instituciones que propendan a instaurar el uso del diálogo como métodos principales de vinculación entre los actores, destinados a la prevención y/o solución de los conflictos, solucionando con ello la problemática la- boral y sentando bases sólidas que ayuden a generar un cambio cultural en la forma de relacionarse y en la capacidad para generar reglas comunes" (p. 8).

Es por ello que las instituciones educativas son y serán el marco de acción y transformación en los estudiantes, al establecer un pensamiento crítico y analítico de la realidad, por lo que es la instancia ideal para inicial un cambio radical en las acciones sobre los conflictos de la comunidad; además de estar conscientes de las consecuencias tan negativas que genera actualmente la violencia a nivel social y personal, que además produce cambios neuronales modificando las conductas del individuo.

La invitación gira entorno a la necesidad de generar profesionistas que tengan las habilidades y herramientas óptimas para la resolución de conflictos, y que, en cualquier ámbito, sean conductos de paz y de diálogo,

los nuevos planteamientos curriculares afirman que todo egresado de la educación obligatoria, debe ser una persona que: "se conozca y respete a sí misma, asuma y valore su identidad, reflexione sobre sus propios actos, conozca sus debilidades y fortalezas, confíe en sus capacidades, sea determinada y perseverante; reconozca como iguales en dignidad y en derechos a todos los seres humanos, y sea empática al relacionarse con otras 
personas y culturas; sepa trabajar en equipo y tenga capacidad de liderazgo; en la solución de conflictos, favorezca el diálogo, la razón y la negociación (Lara Romero, Alamilla y García, 2017, p. 41).

Tener una sociedad preparada para incursionar en los MASC inicia en las instituciones socializadoras como la familia o la escuela: cambiar paradigmas en la primera se da a través de programas sociales, masivos y comunicacionales; sin embargo, la educación formal permitirá obtener nuevas generaciones de personas que en su incursión en el mundo laboral puedan ejercer su función de una manera diferente, aumentando su efectividad, e incluso impactar en la formación de nuevas familias con diferentes formas de educar, comunicarse y resolver sus diferencias.

Para ser mediador, ninguna teoría hasta hoy estudiada sobre los MASC obliga a ser abogado o a tener estudios profesionales, pero sí establece una capacitación mínima sobre el tema, ya que esta persona tendrá la posibilidad de participar e intervenir en la manera de pensar, relacionarse y la toma de decisiones de los intervinientes, por lo que se debe tomar con la seriedad que conlleva, y su instrucción no debe dejarse como algo opcional o de menor importancia, si no como una herramienta básica del profesionista, que en el caso de los abogados ayudará a despresurizar las instancias judiciales en cuanto a la reduc- ción de conflictos que lleguen a la presentación de demandas, pero también la manera de abordar las controversias en todos los ámbitos: tanto público como privado en cualquier tema, ya que se insiste en que los MASC caben en cualquier tema donde se requiera la escucha activa, resoluciones a raíz de un proceso dialógico y la satisfacción mutua de necesidades.

La medición es un tema actual, ya que forma parte de nuestras leyes y como tal, debe ser analizada su pertinencia en cada caso en particular, y lo harán las personas en cada ámbito de participación: en lo laboral, transformará resultados y efectividad de las empresas e instituciones y a su vez, causará un efecto dominó hacia las familias y de las propias instituciones educativas.

Para que esta alternativa funcione, contará con personas especializadas y familiarizadas con el tema, facilitará su elección sobre cualquier otro tipo de solución y ponderación: "La mediación ha destacado en cuanto a su empleo y efectividad en este tiempo en la mayoría de los centros de justicia alternativa de los poderes judiciales locales del país, aunque no en todos, ya que los resultados han sido muy diversos debido a que cada Estado ha desarrollado su propio programa de mediación, y a veces, las diferencias entre unos y otros es muy notable” (Márquez \& De Villa, 2016, p. 49).

$\mathrm{El}$ reto es generar conciencia en las universidades y quienes las dirigen, sobre la pertinencia de la educación de los 
mecanismos alternativos de solución de controversias como una habilidad actual necesaria, que además, es un tema que ya se encuentra en las leyes y reglamentos al que en algún momento se enfrentarán todos los ciudadanos que viven en sociedad, sea en un proceso administrativo, con las autoridades; familiar, como un divorcio; laboral, como una rescisión de contrato; penal, como daños; vecinal con nuestros semejantes, entre otros, que de manera enunciativa mas no limitativa, nos permita entender que en cualquier momento podemos formar parte de un conflicto, pero también de la solución, si tenemos la familiaridad para hacer uso de dichas herramientas.

Que la universidad sea una generadora de nuevos facilitadores posibilitará también la formación de nuevos nichos de trabajo, brindará plusvalía a los futuros profesionistas en los programas de estudio actuales y conciencia en todo ciudadano: comunicación efectiva, conocimiento y autocontrol de emociones y entendimiento de las necesidades del otro, todo como un proceso en dos sentidos: quitar el sentimiento de víctima o injusticia y recuperar la habilidad o potestad de encontrar soluciones efectivas, permanentes y libres.

Los MASC protegen la libertad de las personas, la certeza jurídica de sus instituciones, la confidencialidad y particularidad que cada conflicto alberga, sin etiquetas, expectativas falsas o señalamientos, lo que motiva a que su estudio formal sea parte de la formación del alumno universitario, si se desea que este forme parte de esta sociedad globalizada como agente radical de cambio y transformación en un periodo casi inmediato.

\section{Referencias bibliográficas y fuentes de consulta}

Aguirre, G. (2014, julio-septiembre). TIC y mediación en la enseñanza de la investigación. Razón y palabra. Primera revista electrónica en Iberoamérica especializada en comunicación, (18)87, pp. 1-17. http://www.razonypalabra.org.mx/N/N87/V87/28_Aguirre_V87. pdf

Alonso, P. (2018) La mediación como medio de resolución de conflictos en el ámbito universitario. Tesis doctoral. Facultad de administración y dirección de empresa. Valencia, España. UPV. https://riunet.upv.es/bitstream/ handle/10251/27986/proyecto\%20final.pdf?sequence $=1$

Alzate, R. (2010, octubre) Importancia de la educación en resolución de conflictos. Revista de mediación, 3(6). https://revistademediacion.com/wp-content/uploads/2013/08/ Revista-Mediacion-6-02.pdf

Andrade, Y. (2012, julio-septiembre). La justicia alternativa en México. Una visión a través de los derechos humanos. Ius, Revisa Jurídica. Universidad Latina de América, 42. http:// www.unla.mx/iusunla42/reflexion/LA\%20 JUSTICIA\%20ALTERNATIVA\%20EN\%20 


\section{MEXICO\%20ANDRADE\%20MORALES\%20}

Yurisha.htm

Arias, A., \& Arias, M. (2017). Conflicto y educación superior: narrativas y vivencias de jóvenes universitarios estudiantes de ciencias sociales y humanas. Revista CES Psicología, 11(1), 56-68.

Banco de Desarrollo de América Latina (2016, agosto). Reporte de economía y desarrollo 2016, más habilidades para el trabajo y la vida: los aportes de la familia, la escuela, el entorno y el mundo laboral. Bogotá, Colombia. https://scioteca.caf.com/bitstream/ handle/123456789/936/RED2016-16sep.pdf?sequence $=5$

Cabedo, S. (2001). Pluralidad cultural y convivencia social. Recerca. Revista de pensamiento y análisis, Universidad Jaume-I, España.

Cabello, P. (2013). La mediación como política social. Una vía eficaz para el logro de la cultura de paz. Mediaciones sociales, 12, 191-214.

Cámara de Comercio de España y Programa de Justicia Civil de la Unión Europea (s.f.). Procedimientos de mediación concentrados en un marco judicial. https://www.camara.es/ sites/default/files/2015-10 26_manual_procedimientos_mediacion.pdf

Caycedo, R. \& Cocunubo, N. (2016) La mediación como una solución alternativa de la violencia escolar. Investigaciones Andina, 18(33), 1729-1749. https://www.redalyc.org/ $\mathrm{html} / 2390 / 239053104009 /$

Congreso del Estado de Chihuahua Número 73 (2015, 30 de mayo). Ley de Justicia Alternativa del Estado de Chihuahua. Periódico Ofi- cial del Estado de Chihuahua, Chihuahua, México.

Constitución Política de los Estados Unidos Mexicanos (2019, 26 de marzo). Artículo 3, 17. Última reforma publicada en Diario Oficial de la Federación, México.

Cornelio, E. (2014, junio). Los mecanismos alternativos de solución de controversias como derecho humano. Barataria, Revista castellano-manchega de Ciencias Sociales, 17, 81-95. https://www.redalyc.org/ pdf/3221/322132552006.pdf

Criado, A. (2010, marzo). Espacio abierto. Los mediadores no existimos. Revista de Mediación, 3(5), 1-7. https://revistademediacion. com/wp-content/uploads/2013/06/Revista-Mediacion-05-02.pdf

Díaz Álvarez, R. (2016). La calidad de la mediación en Nuevo León, México. Universidad de Murcia, 1-276.

Fernández, M. P. (2017, julio). Los mecanismos de solución de conflictos: una visión alternativa en la impartición de justicia. Divulgare. Boletín de la Universidad Autónoma del Estado de Hidalgo, (4)8.

Gómez, A. I. (2014). Conciliación y mediación en el derecho del trabajo. Temas selectos de derecho laboral. Instituto de investigaciones jurídicas, Universidad Nacional Autónoma de México, 123-139. https://archivos.juridicas.unam.mx/www/bjv/libros/8/3809/7.pdf Gómez, G. (2013). Conflicto en las organizaciones y mediación. Málaga, España: Universidad Internacional de Andalucía. 
https://dspace.unia.es/bitstream/handle/10334/2558/0477_GomezFunes.pdf

González, N. (2014). El ABC de la mediación en México. Instituto de Investigaciones Jurídicas, Universidad Nacional Autónoma de México, 1-36. http://www.asadip.org/v2/ wp-content/uploads/2013/12/NURIA-El-ABCde-la-mediacion-en-Mexico.pdf

Grau, S., González, C., \& Álvarez, J. (2016). La mediación universitaria: Un recurso de orientación. Experiencia en la Universidad de Alicante. INFAD Revista de psicología, (16)1, 365-378. https://rua.ua.es/dspace/bitstream/10045/56608/1/2016_Grau_etal_INFAD.pdf

Herrera, H. (2008). La mediación como herramienta para evitar los procesos laborales. Tohil. Revista universitaria de la facultad de derecho, 23, 1-21. http://www.derecho.uady. $\mathrm{mx} /$ tohil/rev23/mediacion.pdf

Inciarte, N., \& González, L. (2009). Competencias del docente de educación superior como mediador en los procesos de investigación y evaluación de los aprendizajes. Omnia, 15(2), 39-55.

Jiménez Fernández, A. (2008). Guía didáctica para formación de mediadores en el I.E.S. María Galiana. http://eoepsabi.educa.aragon. es/descargas/H_Recursos/h_1_Psicol_Educacion/h_1.8.Mediacion/09.Formacion_de_ mediadores.pdf

Junco, I. (2010, septiembre). Conflictos y estrategias de mediación en la escuela. Temas para la educación, Revista digital para profe- sionales de la enseñanza, 10, 1-9. https://www. feandalucia.ccoo.es/docu/p5sd7442.pdf

Lara Romero, L., Alamilla García, M., García Hernández, J. (2017). Mediación escolar en el Modelo Educativo para la Educación Obligatoria en México. International Journal of Humanities and Social Science Invention, 6(7), 37-42.

Lungman, S. (1996). La mediación escolar. Buenos Aires: Lugar Editorial.

Macho, C. (2014). Origen y evolución de la mediación: El nacimiento del "movimiento ADR" en Estados Unidos y su expansión a Europa. Anuario de Derecho Civil, 67(3), 931996. https://dialnet.unirioja.es/servlet/articulo?codigo $=4824884$

Márquez, M. \& De Villa, J. (2016, 27 de enero). Mediación y participación ciudadana en México. Ius Humani. Revista de Derecho, 5, 45-68. Navas Córdoba, J. (2012, marzo 20). El futuro de las misiones de la ONU: El papel de las Organizaciones Regionales. Documento de opinión. Instituto Español de Estudios Estratégicos, 88, 1-18. http://www.ieee.es/Galerias/fichero/docs_opinion/2012/DIEEEO25-2012_ FuturoMisionesONU_JulioNavas.pdf

Novel Martí, G. (2010). Mediación Organizacional: Desarrollando un modelo de éxito compartido. Madrid, España: Instituto Complutense de mediación y gestión de conflictos.

Organización de las Naciones Unidas (1999). Declaración y Programa de Acción sobre una Cultura de Paz. Resolución A RES/ 53/243. Resoluciones Aprobadas por la Asamblea General. http://www.fund-cultu- 
radepaz.org/spa/DOCUMENTOS/DECLARACIONES,\%20RESOLUCIONES/Declaracion_CulturadPaz.pdf

Ortiz Pérez, J. (2016). Proyecto de intervención escolar mediante la mediación. Tesis de maestría en la Universidad Miguel Hernández, Centro para el estudio y la prevención de la delincuencia. España. http://dspace.umh.es/bitstream/11000/2581/1/Juan\%20Ortiz\%20 P\%C3\%A9rez_590158.pdf

Ortuño, E. (2014). La cultura de la mediación: Impacto de un programa preventivo de sensibilización, en IES de la región de Murcia. Tesis doctoral. Universidad de Murcia. España. https://digitum.um.es/xmlui/bitstream/10201/39406/6/LA\%20CULTURA\%20 DE\%20LA\%20MEDIACI\%C3\%931.pdf

Pantoja, A. (2005). La gestión de conflictos en el aula. Factores determinantes y propuestas de intervención. España: Ministerio de Educación y Ciencia, 1-36. https://www4.ujaen.es/ apantoja/mis_libros/gestion_confli_05.pdf

Paris, S. (2005). La transformación de los conflictos desde la filosofía para la paz. Tesis doctoral en la Universidad Jaume I de Castellón de la plana, España.

Pérez Jiménez, M. (2016). Guía práctica para el desarrollo de competencias en mediadores. Ministerio de Educación de la República Dominicana. https://www.unicef.org/republicadominicana/Guia_Practica_Desarrollo_WEB.pdf

Pesqueira Leal, J. \& Ortiz Aub, A. (2010). Mediación asociativa y cambio social. El arte de lo posible. México: Universidad de Sonora / Editorial Unison.

Pizarro, S. (2018). Calidad y mejora de la educación: Mediación en las instituciones educativas. Tendencias Pedagógicas, 31, 202-225.

Poblete, P. (2012, 12 de septiembre). La mediación laboral y sus ventajas para el Chile de hoy. Tesis pregrado para optar al grado de licenciado en Ciencias Jurídicas y Sociales, Universidad de Chile.

Prieto, F. (2017, 18 de diciembre). La mediación en el ámbito universitario, ¿Podría encajar la Mediación en el ámbito universitario? A mediar News. Granada, España. Licencia bajo Creative Commons Reconocimiento. https:// www.amediar.info/mediacion-en-el-ambito-universitario/

Rodríguez, D. (2017). La mediación en los conflictos interpersonales entre directivos y maestros de las escuelas secundarias técnicas del municipio de Monterrey, Nuevo León. Tesis doctoral de la Universidad Autónoma de Nuevo León, México.

Secretaría de Educación Pública (2015). Marco de referencia sobre la gestión de la convivencia escolar desde la escuela pública. https://www.gob.mx/sep/documentos/marco-de-referencia-sobre-la-gestion-de-la-convivencia-escolar-desde-la-escuela-publica 
